\title{
Effects of a 2-year healthy eating and physical activity intervention for 3-6-year-olds in communities of high and low socio-economic status: the POP (Prevention of Overweight among Pre-school and school children) project
}

\author{
Valerie De Coen ${ }^{1, *}$, Ilse De Bourdeaudhuii ${ }^{2}$, Carine Vereecken ${ }^{1,3}$, Vera Verbestel ${ }^{2}$, \\ Leen Haerens ${ }^{2,3}$, Inge Huybrechts ${ }^{2}$, Wendy Van Lippevelde ${ }^{1}$ and Lea Maes ${ }^{1}$ \\ ${ }^{1}$ Faculty of Medicine and Health Sciences, Department of Public Health, Ghent University, Watersportlaan 2, \\ B-9000 Ghent, Belgium: '2Department of Movement and Sport Sciences, Ghent University, Ghent, Belgium: \\ ${ }^{3}$ Research Foundation Flanders, Brussels, Belgium
}

Submitted 1 July 2011: Final revision received 31 January 2012: Accepted 1 February 2012: First published online 8 March 2012

\begin{abstract}
Objective: The purpose of the present study was to evaluate the effects of a school-based, 2-year, multi-component intervention on BMI, eating and physical activity behaviour in Flanders, Belgium, targeting children aged 3-6 years in communities of high and low socio-economic status (SES).

Design: Cluster-randomized controlled trial.

Setting: Thirty-one pre-primary and primary schools in three different intervention communities and three paired-matched (on SES profile) control communities in Flanders, Belgium.

Subjects: BMI Z-scores at baseline and follow-up were calculated for 1102 children. Questionnaires with sociodemographic data and FFQ were available from 694 of these 1102 children.

Results: No significant effects were found on BMI Z-scores for the total sample. However, there was a significant decrease in BMI $Z$-score of $0 \cdot 11$ in the low-SES intervention community compared with the low-SES control community, where the BMI $Z$-score increased by $0 \cdot 04(F=6 \cdot 26, P=0 \cdot 01)$. No significant intervention effects could be found for eating behaviour, physical activity or screen-time. There were no significant interaction effects of age and gender of the children on the outcome variables

Conclusions: Although no significant effects were found for BMI Z-scores in the total sample, this intervention had a promising effect in the low-SES community of reducing excess weight gain among young children.
\end{abstract}

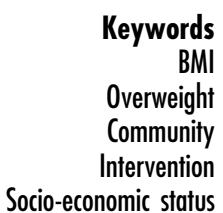

The increasing prevalence of overweight worldwide is an important health issue ${ }^{(1)}$. A number of reports ${ }^{(2,3)}$ show the key role of healthy nutrition and physical activity (PA) in morbidity and mortality in Western nations. Nutrition and PA play an important role in developing overweight and certain risk factors in early childhood. Childhood overweight is a risk factor for adult health $^{(4)}$. The development of lifestyle habits starts early within the child's family. Reviews on prevention of overweight imply interventions should be implemented at a young age ${ }^{(5,6)}$.

Families play an important role in children's development of eating and PA habits. However, families are not isolated entities; they are influenced by a broader environment as defined by the socio-ecological model: family, friends, the community, local policies and the media ${ }^{(7)}$. The physical environment and its effects are well documented, e.g. the availability of safe parks and playgrounds can increase children's $\mathrm{PA}^{(8)}$. Several studies show that adverse socioeconomic characteristics of an area have a negative effect on the health of the population ${ }^{(9)}$ but there is also evidence that social community characteristics, like social capital, have a positive influence on different dimensions of health ${ }^{(10)}$. Tunstall et al. showed that areas with similar adverse economic histories do not all have similarly high mortality rates $^{(11)}$. This leads to the hypothesis that socio-cultural features of areas can play a mediating role in countering the negative effects of socio-economic circumstances. Less is known about the possible interactions between individualand area-level social characteristics. Kohen et $a l^{(12)}$ for example found that a disadvantaged neighbourhood has a significant indirect negative effect on children's behavioural problems through less neighbourhood cohesion and punitive parenting. 
Children spend much time at school. Schools are therefore a natural setting for preventive interventions ${ }^{(13)}$ and are considered a suitable medium to reach parents. Although some school-based programmes have favourable effects on BMI, many have not ${ }^{(14)}$. School interventions have had positive effects on the behaviour of children of lower socio-economic status (SES) if the intervention combined educational and environmental aspects ${ }^{(15)}$. Nevertheless, it remains unclear whether besides parental SES, the SES of the community in which the schools are embedded plays a role in the effect of interventions on BMI, eating behaviour, PA and sedentary behaviour of children. In addition, the age group of 3-6 years is an understudied group.

The purpose of the present study was therefore to examine the effects of a 2-year multi-component intervention in local communities with different socio-economic characteristics on the prevention of overweight among 3-6-year-old children. BMI Z-scores, eating behaviour, PA and screen-time are used as outcome variables to identify effects.

\section{Research methods and procedures}

\section{Study design}

The POP (Prevention of Overweight among Pre-school and school children) project was conducted in six communities. The term 'community' encloses a local authority (town or municipality). The six communities were selected from the research regions in Flanders, Belgium disposed by the Flemish Policy Research Centre for Welfare, Health and Family, which commissioned the research project.

The selection of these six communities out of the research regions was based on five socio-economic characteristics: (i) the number of births in underprivileged families; (ii) the proportion of pupils in primary school with a school delay; (iii) the rate of unemployment; (iv) the number of persons on welfare support; and (v) the number of underprivileged foreigners. High scores on these parameters are indicators of a lower SES profile. If a community scored higher than the Flemish mean on three to five of the abovementioned characteristics, it was labelled as 'low SES'; communities with one or two scores higher than the Flemish mean were labelled as 'medium SES'; and communities with no scores higher than the mean were labelled as 'high SES'. Two communities had a high, two had a medium and two had a low SES profile. From each pair of matched communities the researchers allocated one randomly to the intervention condition. To simplify further interpretation, communities are given a code starting with ' $\mathrm{I}$ ' for intervention or ' $\mathrm{C}$ ' for control. As a result, I-highSES was matched with C-highSES, I-medSES with C-medSES and I-lowSES with C-lowSES.

Recruitment of the participants took place in schools. In Flanders, $98 \%$ of children attend pre-primary school (age 3-5 years) and for children at the age of 6 years, education is compulsory. All pre-primary and primary schools in the six communities were invited to participate in the study. Schools were aware of the fact that they were in an intervention community or in a control community. Participation of the schools was voluntary. Thirtyone of the forty-nine invited pre-primary and primary schools participated (response rate 63\%): eighteen (56\%) in the intervention and thirteen (76\%) in the control communities. The response is shown in the study design flowchart in Fig. 1. Within these schools, all children in pre-primary schools (age 3-5 years) and in the first year of primary schools (age 6 years) were admitted to the study. Of the 3242 eligible children, 1589 participated at baseline. To estimate the necessary sample size per community, the a priori power calculation revealed that for a minimum effect size of $0 \cdot 30(\alpha=0 \cdot 05,1-\beta=0 \cdot 80)$ a sample size of seventy-eight per group was needed.

At baseline (September 2008) and follow-up assessment (April 2010), parents completed a questionnaire and the height and weight of the children were measured. The project was approved by the ethical committee of the Faculty of Medicine and Health Science of Ghent University (EC/2007/570). Parents provided a written consent at the start. It applied to the whole duration of the study, irrespective of their further participation in the questionnaires. This implies that all children with consent were measured at baseline and at follow-up.

\section{Measurements and procedures}

\section{$B M I$ and parental questionnaire}

Height and weight of participating children were measured barefooted and in light clothing in the schools by the research team. Weight was measured to the nearest $0 \cdot 1 \mathrm{~kg}$ (Seca Robusta 813; Seca, Hamburg, Germany) and height to the nearest millimetre using a mobile stadiometer (Seca 214). BMI Z-scores were calculated with Flemish reference data using the LMS (curve-L, mean-M and coefficient of variation- $\left.S^{(16)}\right) \operatorname{method}^{(17)}$.

Demographic factors like birth date, sex and educational level of the parents were collected using a questionnaire filled in by one of the parents. A validated twenty-four-item semi-quantitative $\mathrm{FFQ}^{(18)}$ assessed quantities and frequencies of the consumption of food groups of the intervention's target behaviours: consumption of water, soft drinks, milk, vegetables, fruits, sweets and savoury snacks. The FFQ was validated in a sample of 650 children, aged $2 \cdot 5-6 \cdot 5$ years, using estimated diet records of $3 \mathrm{~d}$ as a reference. Reproducibility ( $n$ 124) was measured by repeated FFQ administrations five weeks apart. For most foods, a moderate level of relative validity was observed for estimation of food intake.

To assess structured PA parents were asked whether their child was a sports club member and for how many hours per week they participated in sports through this club; and whether their child participated in after-school sports 


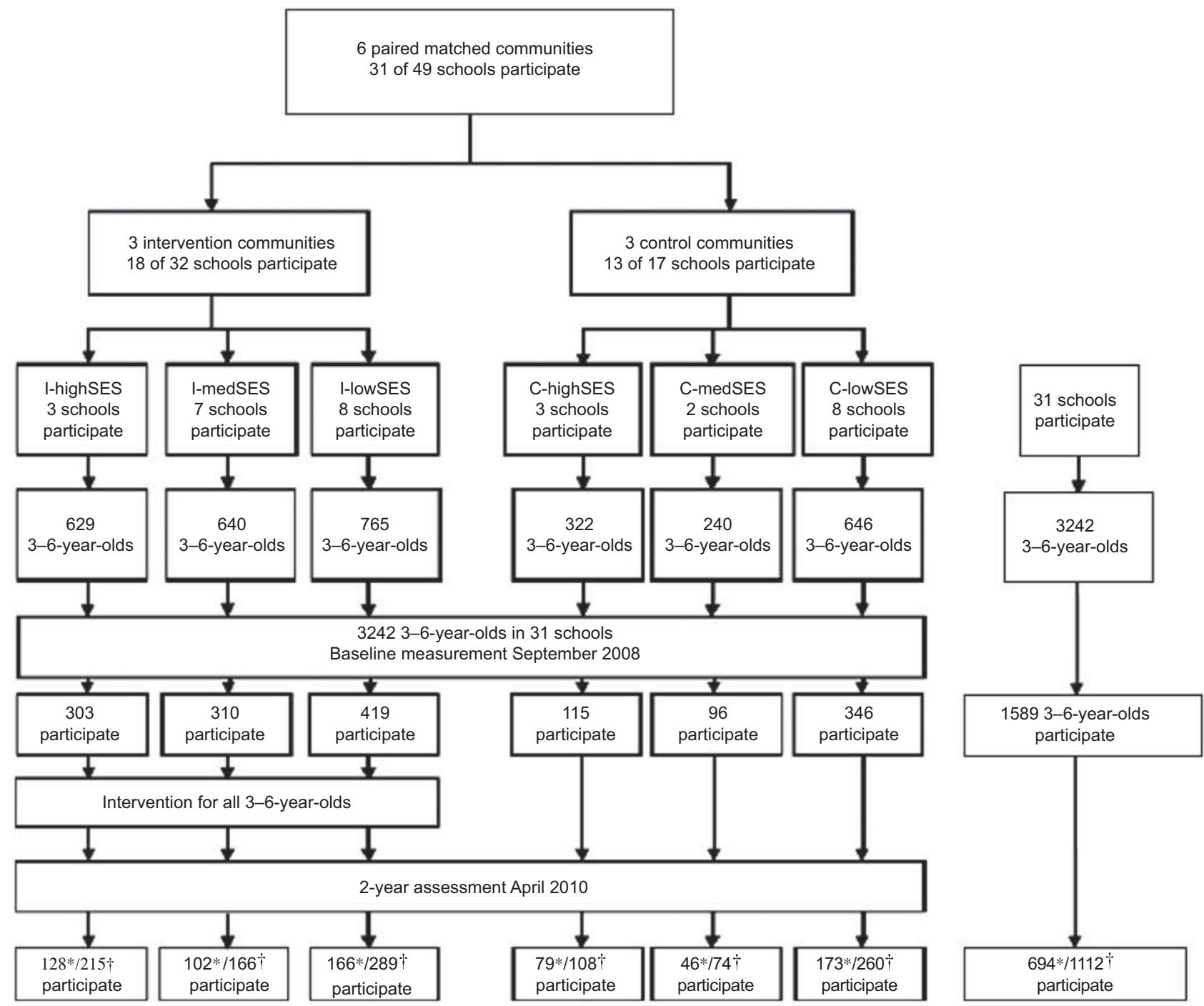

Fig. 1 Flowchart showing the study design and participant response ("participants of the questionnaire, tparticipants at measurement of height and weight)

activities and for how many hours per week. Screen-time behaviour was measured by hours of daily screen-time spent (television, computer, DVD, games console, etc.).

The questionnaire was distributed by teachers in the schools for parents to fill in at home, return to the teachers and be collected by the research team. In some schools, parents experiencing language or other problems filling in the questionnaire were assisted by an intercultural worker or an interpreter.

\section{Community questionnaire}

To have indicators on local nutrition and PA policy, members of the city council were contacted to fill in an online questionnaire assessing nutritional and PA policy, actions and facilities in their community. An existing questionnaire, developed by the Flemish Health Promotion Institute (VIGeZ), the regional health boards and the Flemish Government, called 'Hearty Neighbourhood', was used ${ }^{(19)}$. A health policy profile was generated on three aspects of their policy: (i) nutrition and PA policy; (ii) raising awareness of the population for nutrition and PA; and (iii) expertise in nutrition and PA promotion.

\section{Process evaluation data}

The level of implementation of the seven modules of the school intervention was measured through questionnaires filled in by the teachers.

\section{The intervention}

The intervention was based on the socio-ecological model in health promotion programmes ${ }^{(20)}$ with the child as the centre of focus situated within several layers (family, friends, pre-primary or primary schools, community stakeholders, local policy and media). The intervention was implemented over two school years (2008-2009 and 2009-2010) on different levels. 


\section{Multi-topic intervention}

The intervention was based on the 'Nutrition and Physical Activity Health Targets' of the Flemish Community ${ }^{(21)}$ clustered into: (i) increasing daily consumption of water and decreasing soft drinks consumption; (ii) increasing daily milk consumption; (iii) increasing daily consumption of vegetables and fruit; (iv) decreasing daily consumption of sweets and savoury snacks; and (v) increasing daily PA and decreasing screen-time behaviour.

\section{The community}

At the start, two meetings were held in each intervention community with the researchers, community organizations and stakeholders regarding local social and health problems using concept mapping, a method developed by Trochim ${ }^{(22,23)}$. This method enabled the research team to visualize relevant ideas from the community stakeholders.

Community organizations, members of the city council, aldermen and local non-profit organizations working with children or health topics were approached to support the intervention at community level, to raise awareness and give greater publicity to the project.

Each intervention year, information brochures and posters regarding the five topics of the project were distributed through general practitioners, pharmacists, social services and at relevant community events by the regional health boards and the research team.

\section{The schools}

The schools were the most important setting for the implementation of the intervention. This part was inspired by the Healthy School framework ${ }^{(24)}$ focusing on implementing the topics on the class, the school and the environmental level. The programme consisted of both newly developed and existing materials. To facilitate the implementation, the intervention was described in seven modules. The schools received a manual for each module describing the objectives, the tasks and responsibilities, a suggested timeline and a selection of theory-based methods and practical strategies. These seven modules were: (i) the organization of the POP project at school level; (ii) the organization of classroom activities (Healthy Weeks), including suggested dose and content; (iii) development of an active playground; (iv) implementation of health-related physical education (guidelines by Verstraete et $a l^{(25)}$ ); (v) environmental and policy changes to increase the availability of water at school (e.g. water fountains); (vi) environmental and policy changes to increase to availability of vegetables and fruits at school; and (vii) educational strategies for parents on all topics.

All intervention schools were requested to (i) implement five Healthy Weeks per intervention year (one for each cluster of topics) with a minimum $1 \mathrm{~h}$ of classroom time dedicated to the topic together with extracurricular activities (e.g. during the vegetables and fruits week only fruits could be brought to school as a snack; schools organized fruit and vegetable tastings), (ii) evaluate and improve their playground and snack and beverage policy, and (iii) communicate with the parents on the programme and distribute materials to the parents. The intervention started with a meeting with the teachers during which they received manuals and guidelines and an implementation plan was discussed.

During each intervention year there were three meetings with the teachers to follow up the implementation of the intervention and discuss the possible problems. They received $250 €$ from the research project to buy materials or finance environmental changes.

\section{The parents}

The intervention materials for the parents were newly developed for the project. The parents received a poster visualizing the target messages and containing short tips regarding parenting practices and styles to encourage children to stick to the healthy eating and PA targets. Parents also received five letters, containing detailed information on the intervention topics and a website link with practical information such as tips and recipes. Based on the FFQ in the parental questionnaire, parents received a written, normative individual tailored advice on their child's consumption of water, milk, fruits, vegetables, soft drinks and sweet and savoury snacks, and their PA and screen-time behaviour.

\section{The regional health boards}

The regional health boards are non-profit organizations subsidized by the Flemish Government. They unite local authorities, social services, boards of general practitioners, socio-cultural and welfare organizations and experts to organize and implement local health promotion interventions. The regional health boards supported the schools and community for the implementation of the project. They contacted each school at least twice per year assisting them in selecting relevant intervention materials and supervising the implementation progress. They received $500 €$ for their input in the project.

\section{The research team}

The research team was responsible for measurements, developing the intervention concept, parental materials and for the supervision of the regional health boards. The poster, letters and tailored advice were developed by the research team. They also trained the regional health boards during half a day for their task and organized meetings every three months to discuss progress and problems in the schools and communities.

\section{Statistical analyses}

Data were analysed using the SPSS/PC statistical software package version $15 \cdot 0$ for Windows (SPSS, Inc., Chicago, IL, USA). Statistical power calculations and effect size calculations were done using $G^{*}$ Power $3 \cdot 1 \cdot 3$ (University of Düsseldorf, Düsseldorf, Germany). Descriptive analyses included descriptive statistics of sample characteristics. 
Table 1 Results of the health policy questionnaire

\begin{tabular}{lcccc}
\hline Community & Population & $\begin{array}{c}\text { Nutrition and } \\
\text { PA policy }\end{array}$ & $\begin{array}{c}\text { Raising awareness for } \\
\text { nutrition and PA }\end{array}$ & $\begin{array}{c}\text { Nutrition and PA } \\
\text { promotion expertise }\end{array}$ \\
\hline I-highSES & 12000 & Low & Low & Low \\
C-highSES & 14000 & Medium & Low & Low \\
l-medSES & 30000 & Low & Low & Low \\
C-medSES & 23000 & Medium & Medium & High \\
I-lowSES & 68000 & High & Low & Medium \\
C-lowSES & 35000 & High & Medium & High \\
\hline
\end{tabular}

PA, physical activity; I, intervention; SES, socio-economic status; C, control.

Independent-samples $t$ tests and $\chi^{2}$ analyses were used to assess the differences in characteristics between intervention, control and community samples.

To establish effects of SES, educational level of the mother was used as an indicator for SES. An estimate of SES of the family was obtained by classifying the educational level of the mother as follows: low $=$ highest educational level is higher secondary education; high $=$ highest educational level is bachelor's or master's education.

To assess the effects of the 2-year intervention, linear mixed models were applied on the pre/post difference in BMI $Z$-score and behaviours, entering baseline levels as covariates. A positive change in BMI $Z$-score is an indication for an increase in relative BMI; a negative change indicates a decrease in relative BMI. To prevent bias from outliers, all cases with a BMI Z-score lower than -3 (1st percentile) or higher than +3 (99th percentile) at baseline were not included in the analyses ( $n$ 14) considering a presumption of measurement error cannot be ruled out. First, for BMI $Z$-score, the linear mixed model analyses were applied in the global sample with condition entered as a factor. Next, the community was entered as a possible moderating factor by investigating the interaction between condition and community. As this interaction with community showed a trend for significance $(P=0.085)$, thus supporting our general hypothesis that the intervention had different effects in communities of different SES, separate analyses were repeated per matched communities. Children were clustered in thirty-one schools (nesting variable). $P$ values $\leq 0.05$ were considered statistically significant, values $>0 \cdot 05$ and $\leq 0 \cdot 10$ as a trend for significance. The same method was used to determine effects on behaviour: linear mixed models were used on the difference in the consumption of vegetables, fruits, water, milk, sweet and savoury snacks and weekly/ daily hours of participation in a sports club, after-school sports activities and screen-time. All intervention effects were controlled for parental SES, gender and age.

\section{Results}

\section{Characteristics of the communities}

The population in the communities ranged from 12000 to 68000 inhabitants. The communities differed on nutrition and PA policy, raising awareness for these topics and health promotion expertise. Table 1 shows that I-lowSES scored high on nutrition and PA policy and medium on health promotion expertise, implying that the local policy was aware and active on various health themes but the aspect of raising awareness (low) was lacking. The I-medSES and I-highSES were wealthier than I-lowSES, but had no or a very limited nutrition and PA policy. Overall, of all intervention communities, I-lowSES scored best on nutrition and PA policy profile in spite of their low SES profile.

\section{Sample characteristics and drop-out analyses}

The sample characteristics for the baseline, follow-up and drop-out group are shown in Table 2. At baseline, 1280 parents completed a questionnaire and all children were measured. After the 2-year follow-up, 694 questionnaires were received and 1102 children were measured. This means 586 children dropped out for the questionnaire at follow-up; 178 BMI values could not be attained at follow-up due to absence on the day of the measurement or change of school. The baseline BMI $Z$-scores were not significantly different between the communities. There were no other significant differences between intervention and control regions Over the conditions, participants with a low SES dropped out significantly more at the follow-up measurement $\left(\chi^{2}=10 \cdot 03, P=0 \cdot 001\right)$.

\section{Intervention effects}

Effects of the intervention on BMI Z-score and behaviour are presented in Table 3. No significant effects were found for BMI $Z$-score in the total sample. There was a significant intervention effect for BMI $Z$-score in the lowSES community, I-lowSES $(F=6 \cdot 26 ; P=0 \cdot 01)$, with a decrease in BMI $Z$-score of $0 \cdot 11$ in comparison to an increase of 0.04 in the paired matched control community (C-lowSES). The post hoc power calculation showed that for the effect found in I-lowSES, with 289 respondents in the intervention condition and 260 in the control condition, the observed statistical effect size was 0.46 and the desired statistical power of $0 \cdot 80$ was achieved.

No significant intervention effects could be found for the consumption of fruits, vegetables, water, milk, soft drinks or sweet and savoury snacks, or for participation in a sports club, after-school sports activities or screen-time.

No significant interaction effects between condition and SES on BMI and behaviour were found. This implies 
Table 2 Descriptive characteristics (means, standard deviations and percentages) of the study population: children aged 3-6 years, POP (Prevention of Overweight among Pre-school and school children) project, Flanders, Belgium

\begin{tabular}{|c|c|c|c|c|c|c|c|c|}
\hline & \multicolumn{2}{|c|}{ Age (years) } & \multirow[b]{2}{*}{$\%$ of girls } & \multirow[b]{2}{*}{$\%$ of lower SES } & \multicolumn{2}{|c|}{ BMI $\left(\mathrm{kg} / \mathrm{m}^{2}\right)$} & \multicolumn{2}{|c|}{ BMI Z-score } \\
\hline & Mean & SD & & & Mean & SD & Mean & SD \\
\hline Baseline sample & 4.95 & $1 \cdot 31$ & $50 \cdot 0$ & $32 \cdot 0$ & $15 \cdot 9$ & 1.52 & $0 \cdot 14$ & 0.93 \\
\hline 2-year follow-up & $4 \cdot 98$ & $1 \cdot 30$ & $50 \cdot 1$ & $30 \cdot 9$ & $15 \cdot 9$ & $1 \cdot 44$ & $0 \cdot 12$ & 0.94 \\
\hline Intervention & $4 \cdot 86$ & $1 \cdot 25$ & $47 \cdot 1$ & $33 \cdot 8$ & $15 \cdot 7$ & $1 \cdot 46$ & $0 \cdot 12$ & 0.92 \\
\hline I-highSES & $4 \cdot 91$ & $1 \cdot 11$ & $45 \cdot 3$ & $32 \cdot 8$ & $15 \cdot 9$ & $1 \cdot 65$ & 0.09 & 1.03 \\
\hline I-medSES & $4 \cdot 84$ & $1 \cdot 31$ & $49 \cdot 0$ & $33 \cdot 3$ & $15 \cdot 9$ & $1 \cdot 42$ & $0 \cdot 17$ & 0.88 \\
\hline I-lowSES & $4 \cdot 84$ & $1 \cdot 33$ & $47 \cdot 9$ & $33 \cdot 7$ & $15 \cdot 9$ & $1 \cdot 42$ & $0 \cdot 11$ & 0.92 \\
\hline Control & $5 \cdot 04$ & $1 \cdot 29$ & $54 \cdot 7$ & $29 \cdot 1$ & $15 \cdot 9$ & $1 \cdot 37$ & $0 \cdot 15$ & 0.93 \\
\hline C-highSES & $4 \cdot 94$ & $1 \cdot 17$ & $51 \cdot 9$ & $14 \cdot 1$ & $15 \cdot 9$ & $1 \cdot 35$ & 0.21 & 0.87 \\
\hline C-medSES & $5 \cdot 08$ & 1.54 & $57 \cdot 8$ & $35 \cdot 5$ & $15 \cdot 8$ & $1 \cdot 31$ & 0.09 & 0.89 \\
\hline C-lowSES & $5 \cdot 06$ & $1 \cdot 27$ & $54 \cdot 9$ & $33 \cdot 6$ & $15 \cdot 9$ & $1 \cdot 38$ & $0 \cdot 12$ & 0.95 \\
\hline Drop-outs & $5 \cdot 05$ & $1 \cdot 34$ & $48 \cdot 3$ & 34.6 & $15 \cdot 9$ & $1 \cdot 36$ & $0 \cdot 18$ & 0.89 \\
\hline
\end{tabular}

SES, socio-economic status.

that there is no significant differential effect depending on SES. There were no significant interaction effects of age and gender of the children on the outcome variables.

\section{Discussion}

The main goal of the present study was to evaluate the 2-year effects of an intervention promoting healthy eating and PA on BMI and behaviours in children aged 3-6 years at baseline in communities with different SES profiles. After 2 years of intervention, positive effects were found on BMI $Z$-scores in the low-SES intervention community. BMI Z-score decreased significantly compared with the low-SES control community, where it even increased. No effects on behaviours were measured.

Contrary to what is often done, in the current study place-level and individual-level factors were not separated. Until now, the view of the tight relationships between characteristics of persons and their context has not been fully tested in community intervention studies. Designs of community intervention studies either randomized a limited number of intervention and control communities or implemented interventions in one specific community and matched a similar control community.

Positive effects on BMI of interventions in low-SES communities were reported earlier by Muckelbauer et al. (26) $^{\text {. }}$ where the environmental and educational, school-based intervention on water consumption proved effective in the prevention of overweight among children in primary schools in socially deprived areas. The positive results of recent studies in low-SES communities indicate that community SES might interfere with intervention effectiveness. In many previous intervention studies the context of the intervention was not taken into account and the effects in communities with different SES characteristics were not reported. However, the importance of 'place' has been advocated for a long time by geographers and sociologists, who argue that 'place' is relevant for health variation because it constitutes as well as contains social relationships and physical resources ${ }^{(27)}$. This makes it clear that more attention should be given to characteristics of the context in which interventions are implemented.

Apart from the community's social aspects, other community characteristics of the community included in the present study might be important in explaining the results. Although the community questionnaire indicated that both the low-SES intervention community and the lowSES control community scored high on nutrition and PA policy, the low-SES intervention community scored low on awareness raising $v$. medium in the low-SES control community. The POP project enhanced considerably awareness raising in this intervention community, as well as through the schools and other organizations. It might be that the combination of a good nutrition and PA policy together with an intensive, relatively personal communication strategy leads to an intervention effect in low-SES communities. In the medium- and high-SES communities there was not an elaborated nutrition and PA policy nor were there specific awareness raising interventions.

Process evaluation data revealed that all schools implemented the requested classroom hour. Regarding the snack and playground policy, it was clear that the requested adjustments asked for more time investment and at the time of observation, most schools did not yet meet up to the standard.

No intervention effects were found on the key behaviours of the intervention in the total study population. An explanation for the lack of effects on behaviours could be measurement issues in this young age group. As there was an effect on BMI $Z$-score, this implies the possibility that children did eat more healthfully, were more physically active and/or less sedentary but that parents were perhaps not able to acknowledge and adequately assess this change. It is possible that the schools had an impact on the children's consumption during the school hours but that parents did not know and report this change. Furthermore, the failure to 
Table 3 Effects of the intervention on BMI Z-score, diet and PA among children aged 3-6 years, POP (Prevention of Overweight among Pre-school and school children) project, Flanders, Belgium

\begin{tabular}{|c|c|c|c|c|c|c|c|c|c|}
\hline & \multicolumn{4}{|c|}{ Intervention } & \multicolumn{4}{|c|}{ Control } & \multirow{3}{*}{$\frac{2 \text {-year effects }}{F}$} \\
\hline & \multicolumn{2}{|c|}{ Pre } & \multicolumn{2}{|c|}{ 2-year post } & \multicolumn{2}{|c|}{ Pre } & \multicolumn{2}{|c|}{ 2-year post } & \\
\hline & Mean & SD & Mean & SD & Mean & SD & Mean & SD & \\
\hline \multicolumn{10}{|l|}{ BMI Z-score } \\
\hline Total group & $0 \cdot 12$ & 0.95 & $0 \cdot 11$ & $1 \cdot 03$ & $0 \cdot 13$ & 0.92 & $0 \cdot 15$ & $0 \cdot 89$ & NS \\
\hline High SES & 0.09 & $1 \cdot 03$ & $0 \cdot 17$ & 0.95 & $0 \cdot 18$ & $0 \cdot 86$ & $0 \cdot 15$ & 0.97 & NS \\
\hline Medium SES & $0 \cdot 17$ & $0 \cdot 88$ & 0.23 & 0.95 & 0.09 & $0 \cdot 89$ & $0 \cdot 11$ & $0 \cdot 83$ & NS \\
\hline Low SES & $0 \cdot 11$ & 0.92 & 0.00 & $1 \cdot 11$ & $0 \cdot 12$ & 0.95 & $0 \cdot 16$ & $0 \cdot 88$ & $6 \cdot 26^{\star \star}$ \\
\hline \multicolumn{10}{|c|}{ Fruit consumption (g/d) } \\
\hline Total group & 142 & $95 \cdot 7$ & 151 & $97 \cdot 4$ & 135 & $84 \cdot 3$ & 139 & $95 \cdot 2$ & NS \\
\hline High SES & 144 & 104 & 164 & $95 \cdot 7$ & 148 & $70 \cdot 9$ & 156 & $94 \cdot 7$ & NS \\
\hline Medium SES & 126 & $82 \cdot 3$ & 135 & $87 \cdot 0$ & 133 & $86 \cdot 4$ & 136 & $94 \cdot 4$ & NS \\
\hline Low SES & 151 & $96 \cdot 7$ & 153 & $93 \cdot 1$ & 130 & $87 \cdot 9$ & 135 & $96 \cdot 9$ & NS \\
\hline \multicolumn{10}{|c|}{ Vegetable consumption $(\mathrm{g} / \mathrm{d})$} \\
\hline Total group & $81 \cdot 8$ & $62 \cdot 5$ & $91 \cdot 2$ & $62 \cdot 6$ & $73 \cdot 2$ & $60 \cdot 6$ & $83 \cdot 3$ & $57 \cdot 5$ & NS \\
\hline High SES & $80 \cdot 7$ & $57 \cdot 6$ & 101 & $61 \cdot 1$ & $76 \cdot 1$ & $49 \cdot 7$ & 101 & $57 \cdot 7$ & NS \\
\hline Medium SES & $76 \cdot 2$ & $57 \cdot 7$ & $87 \cdot 8$ & $50 \cdot 7$ & $77 \cdot 6$ & $63 \cdot 2$ & $86 \cdot 0$ & $56 \cdot 1$ & NS \\
\hline Low SES & $86 \cdot 7$ & $68 \cdot 9$ & $94 \cdot 6$ & $71 \cdot 6$ & $71 \cdot 0$ & $64 \cdot 1$ & $76 \cdot 1$ & $56 \cdot 1$ & NS \\
\hline \multicolumn{10}{|c|}{ Milk consumption $(\mathrm{ml} / \mathrm{d})$} \\
\hline Total group & 357 & 216 & 340 & 243 & 341 & 206 & 315 & 189 & NS \\
\hline High SES & 358 & 209 & 309 & 211 & 324 & 177 & 299 & 175 & NS \\
\hline Medium SES & 324 & 198 & 302 & 191 & 371 & 227 & 352 & 201 & NS \\
\hline Low SES & 379 & 231 & 337 & 197 & 339 & 210 & 313 & 190 & NS \\
\hline \multicolumn{10}{|c|}{ Water consumption $(\mathrm{ml} / \mathrm{d})$} \\
\hline Total group & 280 & 166 & 327 & 168 & 286 & 166 & 315 & 164 & NS \\
\hline High SES & 292 & 159 & 364 & 153 & 277 & 162 & 311 & 170 & NS \\
\hline Medium SES & 278 & 161 & 319 & 164 & 304 & 168 & 290 & 177 & NS \\
\hline Low SES & 274 & 177 & 318 & 172 & 285 & 167 & 318 & 166 & NS \\
\hline \multicolumn{10}{|c|}{ Soft drinks consumption (ml/d) } \\
\hline Total group & $50 \cdot 3$ & $86 \cdot 1$ & $53 \cdot 7$ & $90 \cdot 8$ & $64 \cdot 4$ & 100 & $58 \cdot 2$ & $86 \cdot 1$ & NS \\
\hline High SES & $45 \cdot 9$ & $82 \cdot 7$ & $44 \cdot 1$ & $75 \cdot 5$ & $44 \cdot 9$ & 86.9 & $31 \cdot 9$ & $52 \cdot 7$ & NS \\
\hline Medium SES & $52 \cdot 5$ & $83 \cdot 4$ & $48 \cdot 4$ & $78 \cdot 7$ & $88 \cdot 2$ & 112 & $94 \cdot 4$ & 106 & NS \\
\hline Low SES & $52 \cdot 7$ & $91 \cdot 6$ & $58 \cdot 5$ & $96 \cdot 8$ & $66 \cdot 4$ & 101 & $56 \cdot 0$ & $82 \cdot 3$ & NS \\
\hline \multicolumn{10}{|c|}{ Sweet and savoury snacks consumption (g/d) } \\
\hline Total group & $49 \cdot 9$ & $34 \cdot 4$ & $50 \cdot 0$ & $33 \cdot 0$ & $54 \cdot 8$ & $444 \cdot 9$ & $51 \cdot 1$ & $33 \cdot 5$ & NS \\
\hline High SES & $53 \cdot 5$ & $37 \cdot 5$ & $52 \cdot 3$ & $32 \cdot 4$ & $52 \cdot 3$ & $29 \cdot 9$ & $49 \cdot 8$ & $24 \cdot 7$ & NS \\
\hline Medium SES & $52 \cdot 9$ & $37 \cdot 3$ & $45 \cdot 9$ & $22 \cdot 7$ & $53 \cdot 9$ & $65 \cdot 7$ & $55 \cdot 8$ & $33 \cdot 6$ & NS \\
\hline Low SES & $44 \cdot 9$ & $29 \cdot 6$ & $46 \cdot 4$ & $26 \cdot 3$ & $56 \cdot 2$ & $42 \cdot 9$ & $54 \cdot 0$ & $39 \cdot 4$ & NS \\
\hline \multicolumn{10}{|l|}{ Screen-time $(\mathrm{h} / \mathrm{d})$} \\
\hline Total group & $1 \cdot 4$ & 0.9 & $1 \cdot 4$ & 0.9 & $1 \cdot 3$ & $1 \cdot 1$ & $1 \cdot 4$ & 0.9 & NS \\
\hline High SES & $1 \cdot 5$ & 0.9 & $1 \cdot 6$ & $1 \cdot 0$ & $1 \cdot 2$ & $0 \cdot 8$ & $1 \cdot 3$ & $0 \cdot 8$ & NS \\
\hline Medium SES & $1 \cdot 2$ & $0 \cdot 8$ & $1 \cdot 2$ & $0 \cdot 6$ & $1 \cdot 8$ & $1 \cdot 0$ & 1.9 & $1 \cdot 1$ & NS \\
\hline Low SES & 1.5 & $1 \cdot 0$ & $1 \cdot 5$ & $1 \cdot 0$ & $1 \cdot 4$ & $1 \cdot 1$ & $1 \cdot 6$ & $1 \cdot 0$ & NS \\
\hline \multicolumn{10}{|c|}{ Participation in a sports club (h/week) } \\
\hline Total group & $2 \cdot 32$ & $2 \cdot 38$ & $2 \cdot 08$ & $1 \cdot 66$ & $2 \cdot 15$ & $1 \cdot 86$ & $2 \cdot 49$ & $2 \cdot 27$ & NS \\
\hline High SES & $2 \cdot 90$ & 3.29 & $2 \cdot 07$ & 1.99 & $2 \cdot 67$ & $1 \cdot 79$ & $3 \cdot 80$ & 3.23 & NS \\
\hline Medium SES & 1.53 & 1.69 & $1 \cdot 74$ & $1 \cdot 44$ & $1 \cdot 89$ & $1 \cdot 37$ & $1 \cdot 76$ & $1 \cdot 38$ & NS \\
\hline Low SES & $2 \cdot 27$ & $2 \cdot 11$ & $2 \cdot 24$ & $1 \cdot 48$ & $2 \cdot 19$ & $2 \cdot 16$ & $2 \cdot 64$ & $2 \cdot 30$ & NS \\
\hline \multicolumn{10}{|c|}{ Participation in after-school sports activities (h/week) } \\
\hline Total group & $2 \cdot 05$ & $1 \cdot 16$ & $1 \cdot 48$ & $1 \cdot 76$ & $2 \cdot 09$ & 1.55 & 1.58 & $1 \cdot 76$ & NS \\
\hline High SES & $2 \cdot 37$ & $1 \cdot 08$ & $1 \cdot 17$ & 1.85 & $2 \cdot 60$ & $1 \cdot 79$ & 1.50 & $2 \cdot 26$ & NS \\
\hline Medium SES & $1 \cdot 79$ & 0.96 & $1 \cdot 32$ & $1 \cdot 63$ & 1.59 & $1 \cdot 27$ & $1 \cdot 43$ & $1 \cdot 31$ & NS \\
\hline Low SES & $2 \cdot 02$ & $1 \cdot 25$ & $1 \cdot 69$ & $1 \cdot 77$ & $2 \cdot 15$ & $1 \cdot 55$ & $1 \cdot 67$ & $1 \cdot 79$ & NS \\
\hline
\end{tabular}

PA, physical activity; SES, socio-economic status.

${ }^{\star \star} P \leq 0 \cdot 01$.

observe intervention effects on behaviours might be a consequence of measurement methods that were too crude, or changes which were too small to detect. For example, Muckelbauer et $a l^{(28)}$ found an effect of a combined environmental and educational intervention on water consumption with water consumption defined as number of glasses (with 1 glass defined as $200 \mathrm{ml}$ ) evaluated by a $24 \mathrm{~h}$ recall questionnaire.
There were no intervention effects for hours of participation in a sports club or after-school sports activities. The intervention focused on PA at school and home and on active play time and reducing screen-time or sedentary time. Although sports club membership and after-school sports participation are not complete markers of PA behaviour, the other activity measures were subject to strong over-reporting, not valid, and not included in the 
analyses. The same problem might have occurred with the reported screen-time by parents.

The prevention of overweight is an important health issue today. The present study showed there is good reason to believe that socio-economic characteristics of the community significantly influence an intervention on prevention of overweight in young children. Our findings suggest that it might be worthwhile to take regional community characteristics into account when evaluating interventions.

\section{Strengths and limitations}

The strength of the study is that it examined the effects of an intervention in different communities regarding socio-economic characteristics. It showed that the effects of an intervention for prevention of childhood overweight mainly implemented through schools differed under different local socio-economic characteristics.

However, some study limitations should be considered when interpreting our results. As in many intervention studies, selective drop-out may have influenced the outcomes. Although this was not particular for one condition, the low-SES participants dropped out significantly more. Second, the study included a limited number of communities. Third, there was a larger sample available for the analyses on BMI compared with the questionnaires. Fourth, although the BMI $Z$-score of one community showed some changes, generally there were no changes found in the behaviour to support this, implying the possibility that the measurement method adopted was too crude. Last, the intervention allowed alterations by the schools, community and partners of the project. Given the nature of the present intervention study, a full implementation of the CONSORT statement $^{(29)}$ was not possible; but since it was a quasiexperimental design, we did meet up to the standards of the TREND statement ${ }^{(30)}$. Although practitioners consider this as normal practice, this might be considered as a limitation of the study.

\section{Recommendations}

The current study showed that regional differences can play a role in interventions on the prevention of childhood overweight. However, a study with more different communities is needed for multilevel analysis to confirm these findings and obtain more insight into the mechanisms of local SES characteristics, the local health policy, awareness and local health promotion expertise and their effects on health interventions. A mediation analysis is recommended for further exploration of the role of the community characteristics in intervention effects on healthy nutrition and PA. Designing and evaluating interventions that can benefit from the local community characteristics is a challenge for the future but as stated by Moller $^{(31)}$, it is rare to find a situation with enough funding, means, expertise and participating communities available to use accepted methods and standardized interventions to obtain reliable and qualitative results. There is a need for research methods allowing an assessment of mixed strategies and populations. Debate on the methods of measurement and process evaluations should be encouraged and the development of new methods is advisable.

\section{Acknowledgements}

The study was commissioned, financed and steered by the Ministry of the Flemish Community (Department of Economics, Science and Innovation; Department of Welfare, Public Health and Family). The work was performed by the Centre of Expertise for Welfare, Public Health and Family, which is a consortium of researchers of the Catholic University of Leuven, Ghent University, Vrije Universiteit Brussel and Katholieke Hogeschool Kempen. C.V. and L.H. are postdoctoral researchers funded by the Research Foundation Flanders. There were no conflicts of interest. The work presented here was carried out in collaboration between all authors. V.D.C., I.D.B., L.M., V.V. and C.V. defined the research theme. V.D.C., I.D.B., L.M. and C.V. designed methods and experiments, carried out the analyses and interpreted the results. L.H., I.H. and W.V.L. discussed analyses, interpretation and presentation. All authors have contributed to, seen and approved the manuscript.

\section{References}

1. Wang Y \& Lobstein T (2006) Worldwide trends in childhood overweight and obesity. Int J Pediatr Obes 1, 11-25.

2. World Health Organization (2003) The First Action Plan for Food and Nutrition Policy. Copenhagen: WHO Regional Office for Europe.

3. World Health Organization (2004) Global Strategy on Diet, Physical Activity and Health. Copenhagen: WHO Regional Office for Europe.

4. Reilly JJ \& Kelly J (2011) Long-term impact of overweight and obesity in childhood and adolescence on morbidity and premature mortality in adulthood: systematic review. Int J Obes (Lond) 35, 891-898.

5. Summerbell CD, Waters E, Edmunds LD et al. (2005) Interventions for preventing obesity in children. Cochrane Database Syst Rev issue 3, CD001871.

6. Ells LJ, Campbell K, Lidstone J et al. (2005) Prevention of childhood obesity. Best Pract Res Clin Endocrinol Metab 19, 441-454.

7. Booth SL, Sallis JF, Ritenbaugh C et al. (2001) Environmental and societal factors affect food choice and physical activity: rationale, influences, and leverage points. Nutr Rev 59, 3 Pt 2, S21-S39.

8. Roux AVD (2001) Investigating neighborhood and area effects on health. Am J Public Health 91, 1783-1789.

9. Mitchell R, Gleave S, Bartley M et al. (2000) Do attitude and area influence health? A multilevel approach to health inequalities. Health Place 6, 67-79.

10. Kawachi I, Kennedy BP \& Glass R (1999) Social capital and self-rated health: a contextual analysis. Am J Public Health 89, 1187-1193. 
11. Tunstall HVZ, Shaw M \& Dorling D (2004) Places and health. J Epidemiol Commun Health 58, 6-10.

12. Kohen DE, Leventhal T, Dahinten VS et al. (2008) Neighborhood disadvantage: pathways of effects for young children. Child Dev 79, 156-169.

13. Foster GD, Sherman S, Borradaile KE et al. (2008) A policybased school intervention to prevent overweight and obesity. Pediatrics 121, E794-E802.

14. Doak CM, Visscher TLS, Renders CM et al. (2006) The prevention of overweight and obesity in children and adolescents: a review of interventions and programmes. Obes Rev 7, 111-136.

15. De Bourdeaudhuij I, Simon C, De Meester F et al. (2011) Are physical activity interventions equally effective in adolescents of low and high socio-economic status (SES): results from the European Teenage project. Health Educ Res 26, 119-130.

16. Cole TJ (1990) The Lms method for constructing normalized growth standards. Eur J Clin Nutr 44, 45-60.

17. Roelants M, Hauspie R \& Hoppenbrouwers K (2009) References for growth and pubertal development from birth to 21 years in Flanders, Belgium. Ann Hum Biol36, 680-694.

18. Huybrechts I, De Backer G, De Bacquer D et al. (2009) Relative validity and reproducibility of a food-frequency questionnaire for estimating food intakes among Flemish preschoolers. Int J Environ Res Public Health 6, 382-399.

19. VIGez (2008) Hartelijke Buurt. http://www.hartelijkebuurt.be (accessed December 2010).

20. Stokols D (1996) Translating social ecological theory into guidelines for community health promotion. Am J Health Promot 10, 282-298.

21. Zorg en Gezondheid (2008) Vlaams agentschap zorg en gezondheid. http://www.Zorgengezondheid.be (accessed December 2010).
22. Trochim WMK (1989) An introduction to concept mapping for planning and evaluation. Eval Program Plann 12, $1-16$.

23. Kelly CM, Baker EA, Brownson RC et al. (2007) Translating research into practice: using concept mapping to determine locally relevant intervention strategies to increase physical activity. Eval Program Plann 30, 282-293.

24. VIGez (2008) Gezonde school. http://www.gezondeschool. be (accessed December 2009).

25. Verstraete S, De Bourdeaudhuij I, De Clercq D et al. (2006) Meer en beter bewegen. Binnen en buiten de school. Publicatiefonds voor Lichamelijke Opvoeding. Gent: Steunpunt Sport, Beweging en Gezondheid ism Universiteit Gent.

26. Muckelbauer R, Libuda L, Clausen K et al. (2009) A simple dietary intervention in the school setting decreased incidence of overweight in children. Obes Facts 2, 282-285.

27. Macintyre S, Maciver S \& Sooman A (1993) Area, class and health - should we be focusing on places or people? J Soc Policy 22, 213-234.

28. Muckelbauer R, Libuda L, Clausen K et al. (2009) Promotion and provision of drinking water in schools for overweight prevention: randomized, controlled cluster trial. Pediatrics 123, 661-667.

29. Moher D, Schulz KF \& Altman DG (2001) The CONSORT statement: revised recommendations for improving the quality of reports of parallel group randomized trials. $B M C$ Med Res Methodol 1, 2.

30. Des Jarlais DC, Lyles C, Crepaz N et al. (2004) Improving the reporting qualilty on nonrandomized evaluations of behavioral and public health interventions: the TREND statement. Am J Public Health 94, 361-365.

31. Moller J (2004) Reconsidering community based interventions. Inj Prev 10, 2-3. 